\title{
Boomerang Distinguishers on MD4-Family: First Practical Results on Full 5-Pass HAVAL
}

\author{
Yu Sasaki \\ NTT Information Sharing Platform Laboratories, NTT Corporation \\ 3-9-11 Midori-cho, Musashino-shi, Tokyo 180-8585 Japan \\ sasaki.yu@lab.ntt.co.jp
}

\begin{abstract}
In this paper, we study a boomerang attack approach on MD4-based hash functions, and present a practical 4-sum distinguisher against the compression function of the full 5-pass HAVAL. Our approach is based on the previous work by Kim et al., which proposed the boomerang distinguisher on the encryption mode of MD4, MD5, and HAVAL in the related-key setting. Firstly, we prove that the differential path for 5-pass HAVAL used in the previous boomerang distinguisher contains a critical flaw and thus the attack cannot work. We then search for new differential paths. Finally, by using the new paths, we mount the distinguisher on the compression function of the full 5-pass HAVAL which generates a 4-sum quartet with a complexity of approximately $2^{11}$ compression function computations. As far as we know, this is the first result on the full compression function of 5-pass HAVAL that can be computed in practice. We also point out that the 4-sum distinguisher can also be constructed for other MD4-based hash functions such as MD5, 3-pass HAVAL, and 4-pass HAVAL. Our attacks are implemented on a $\mathrm{PC}$ and we present a generated 4-sum quartet for each attack target.
\end{abstract}

Keywords: boomerang attack, 4-sum distinguisher, hash, HAVAL.

\section{Introduction}

Hash functions are taking important roles in various aspects of the cryptography. After the breakthrough by Wang et al. [2627] and through the SHA-3 competition 20], cryptanalysis against hash functions have been improved significantly.

The boomerang attack, which was proposed by Wagner [22], is a tool for the cryptanalysis against block-ciphers. At FSE2011, Biryukov et al. applied the boomerang attack for hash functions, and showed that a zero-sum distinguisher could be constructed on them [3], where zero-sum is a set of messages whose $\mathrm{XOR}$ is 0 and the XOR of their corresponding outputs is also 0. Lamberger and Mendel independently applied the boomerang attack on SHA-2 and obtained a significant improvement on the 4-sum distinguisher against its reduced-step compression function [10, where a $k$-sum is a set of $k$ paired initial-values and messages such that the XOR of their outputs is 0 . It seems that the boomerang attack is potentially very powerful against hash functions, and thus more investigation is required to understand their impact deeply. Note that at CRYPTO2007, 
Joux and Peyrin proposed an (amplified) boomerang attack for SHA-1 7]. They used the idea of the boomerang attack for the message modification technique in the collision attack, which the purpose is different from our research.

The boomerang attack on hash functions does not always discuss the security as the hash function. As done in 10, it often discusses the security of the compression function or the internal block-cipher. Although they do not impact to the security of the hash function immediately, such analyses are useful from several viewpoints; 1) The progress of the cryptanalysis, in other words, the security margin can be measured, 2) The attack could be used as a tool for different purposes in the future, e.g., a pseudo-collision attack on MD5 [4. 3) The attack on a building-block may invalidate the security proof for the hash function. Specifically, hash functions using the PGV modes tend to have the reduction security by assuming the ideal behavior of the internal block-cipher.

MD4, which was proposed by Rivest in 1990 [13, is a hash function that is used as a base of various hash functions. MD4 has an interesting property in its message expansion. The sketch of its computation is as follows;

- Divide an input message block $M$ into several message words $m_{0}$, $m_{1}, \ldots, m_{N_{S}-1}$.

- Iteratively apply a round function $N_{R}$ times, where the round function consists of $N_{S}$ steps.

- For $N_{S}$ steps in each round, each of $m_{0}$ to $m_{N_{S}-1}$ is used exactly once.

- The order of message words, in other words, the permutation of the messageword index may change for different rounds.

We call this type of the message expansion message-words permutation. MD4, MD5 [14, and HAVAL [32] are examples using the message-words permutation.

MD4, MD5, and HAVAL are now known to be vulnerable against various attacks. For example, Van Rompay et al. found collisions of 3-pass HAVAL in 2003 21, and Wang et al. found collisions of MD4, MD5, and 3-pass HAVAL in 2004 [25 27]. The complexity of collision attacks were optimized to 2 for MD4 [18, $2^{10}$ for MD5 [29], $2^{7}$ for 3-pass HAVAL [1924, $2^{36}$ for 4-pass HAVAL 2831, and $2^{123}$ for 5-pass HAVAL 31, where the unit of the complexity is one computation of the compression function. Note that, only the theoretical result is known for 5-pass HAVAL, and thus real collisions have not been found yet.

Theoretical preimage attacks are also presented. For example, 1611] for MD4, 17] for MD5, 2[16] for 3-pass HAVAL, and [16] for 4-pass HAVAL. For 5 -pass HAVAL, only the attack on 158-steps out of 160-steps is known [15].

Several researchers evaluated the security of the building block for these hash functions. Examples which analyzed full steps are 45] for MD5 and 8930] for HAVAL. Among them, the work by Kim et al. 89., which applied the boomerang attack to distinguish their encryption modes from a random permutation in the related-key setting, is very powerful. They successfully distinguished these encryption modes with $2^{6}$ queries for MD4, $2^{11.6}$ queries for MD5, and $2^{9.6}$ queries for 4-pass HAVAL. These attacks were implemented and an example of the boomerang quartet was presented for MD5. In addition, Kim et al. claimed that 5-pass HAVAL could also be distinguished with $2^{61}$ queries and the attack 
Table 1. Comparison of the attack complexity

\begin{tabular}{|c|c|c|c|c|c|c|c|c|c|c|c|}
\hline \multirow{2}{*}{ Attack } & \multirow[t]{2}{*}{ Target } & \multirow{2}{*}{\multicolumn{2}{|c|}{$\begin{array}{c}\text { MD4 } \\
\text { (Time Ref.) }\end{array}$}} & \multirow{2}{*}{$\begin{array}{r}\text { MI } \\
\text { (Time }\end{array}$} & \multirow{2}{*}{$\begin{array}{l}\text { D5 } \\
\text { Ref.) }\end{array}$} & \multicolumn{2}{|c|}{ HAVAL-3 } & \multicolumn{2}{|c|}{ HAVAL-4 } & \multirow{2}{*}{\multicolumn{2}{|c|}{$\begin{array}{l}\text { HAVAL-5 } \\
\text { (Time Ref.) }\end{array}$}} \\
\hline & & & & & & (Time & Ref.) & (Time & Ref.) & & \\
\hline Collision & Hash Function & 2 & 18 & $2^{10}$ & 29 & $2^{7}$ & \begin{tabular}{|l|l|}
19 & 24 \\
\end{tabular} & $2^{36}$ & \begin{tabular}{|l|l|}
28 & 31 \\
\end{tabular} & $2^{123}$ & 31 \\
\hline Boomerang & Block-Cipher & $2^{6}$ & 9 & $2^{11.6}$ & 9 & & & $2^{9.6}$ & 9 & $2^{61}$ & 9 \\
\hline Boomerang & Compress. Func. & - & & $2^{10}$ & Ours & $2^{4}$ & Ours & $2^{11}$ & Ours & $2^{11}$ & Ours \\
\hline
\end{tabular}

was partially verified by implementing it for reduced-round variants. Note that although Kim et al. pointed out the vulnerability of the MD4-based structure against the boomerang attack, the analysis on 5-pass HAVAL is still infeasible.

\section{Our Contributions}

In this paper, we study the boomerang attack approach on MD4-based hash functions. We use the differential path for the boomerang attack to construct the 4-sum distinguisher on the compression function, while Kim et al. [9] used the boomerang path to distinguish its encryption mode from a random permutation. For both of our approach and the one in [9], the core of the attack is the existence of the differential path suitable for the boomerang attack. However, because the attack scenario is different, the procedure to optimize the attack is quite different. We first collect various techniques for the boomerang attack on hash functions from several papers (mainly [3910]), and summarize the attack framework.

We then revisit the differential path for the boomerang attack against 5-pass HAVAL in [9]. On the contrary to the authors' claim, we prove that the differential path in 9] contains a critical flaw and thus the attack cannot work.

We then search for new differential paths for the boomerang attack and construct the attack procedure optimized for attacking the compression function. Finally, by using the new paths, we mount the distinguisher on the full compression function of 5-pass HAVAL which generates a 4-sum quartet with a complexity of $2^{11}$ compression function computations. The attack complexity is summarized in Table 1. As far as we know, this is the first result on the full 5-pass HAVAL that can be computed in practice. The attack is implemented on a PC and we present a generated 4-sum quartet.

Note that as long as the good boomerang differential path is available, 4-sum distinguishers can be constructed on the compression function. Then, with the differential paths in [9], we attack MD5, 3-pass HAVAL, and 4-pass HAVAL with a complexity of $2^{10}, 2^{4}$ and $2^{11}$ compression function computations, respectively. We present generated 4-sums in Appendix B].

\section{Paper Outline}

We describe the specification of HAVAL and clarify the terminology in Sect. 2. We summarize previous work in Sect. 3. We give a summary of techniques for the boomerang attack on hash functions in Sect. 4. We demonstrate a dedicate attack on 5-pass HAVAL in Sect. 5. Finally, we conclude this paper in Sect. 6. 
Table 2. Word-wise rotation $\phi_{x, y}$ of HAVAL

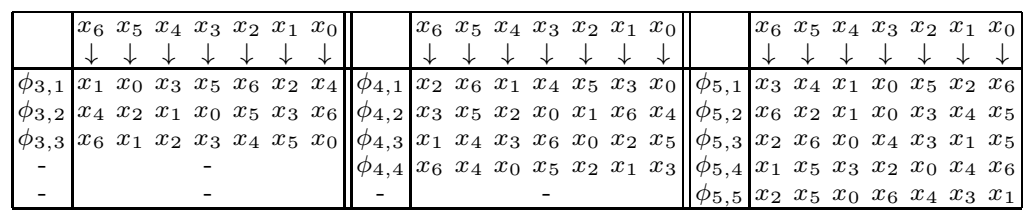

Table 3. Message-words permutation. The first column shows the round numbers.

\begin{tabular}{r|rrrrrrrrrrrrrrrrrrrrrrrrrrrrrrrrrrrrrrrr}
\hline & \multicolumn{11}{c|c}{ index for each round } \\
\hline 1 & 0 & 1 & 2 & 3 & 4 & 5 & 6 & 7 & 8 & 9 & 10 & 11 & 12 & 13 & 14 & 15 & 16 & 17 & 18 & 19 & 20 & 21 & 22 & 23 & 24 & 25 & 26 & 27 & 28 & 29 & 30 & 31 \\
2 & 5 & 14 & 26 & 18 & 11 & 28 & 7 & 16 & 0 & 23 & 20 & 22 & 1 & 10 & 4 & 8 & 30 & 3 & 21 & 9 & 17 & 24 & 29 & 6 & 19 & 12 & 15 & 13 & 2 & 25 & 31 & 27 \\
3 & 19 & 9 & 4 & 20 & 28 & 17 & 8 & 22 & 29 & 14 & 25 & 12 & 24 & 30 & 16 & 26 & 31 & 15 & 7 & 3 & 1 & 0 & 18 & 27 & 13 & 6 & 21 & 10 & 23 & 11 & 5 & 2 \\
4 & 24 & 4 & 0 & 14 & 2 & 7 & 28 & 23 & 26 & 6 & 30 & 20 & 18 & 25 & 19 & 3 & 22 & 11 & 31 & 21 & 8 & 27 & 12 & 9 & 1 & 29 & 5 & 15 & 17 & 10 & 16 & 13 \\
5 & 27 & 3 & 21 & 26 & 17 & 11 & 20 & 29 & 19 & 0 & 12 & 7 & 13 & 8 & 31 & 10 & 5 & 9 & 14 & 30 & 18 & 6 & 28 & 24 & 2 & 23 & 16 & 22 & 4 & 1 & 25 & 15 \\
\hline
\end{tabular}

\section{Preliminaries}

\subsection{Specification of HAVAL}

HAVAL 32] uses a narrow-pipe Merkle-Damgård structure. An input message $M$ is padded to be a multiple of the block-size (1024 bits), and then divided into message blocks $\left(M_{0}, M_{1}, \ldots, M_{L-1}\right)$. Then, chaining variable $H_{i}$ starting from the pre-specified initial value $H_{0}$ is iteratively updated by the compression function $\mathrm{CF} ; H_{i+1} \leftarrow \mathrm{CF}\left(H_{i}, M_{i}\right)$, for $i=0,1, \ldots, L-1$. Finally, $H_{L}$ is the hash value of $M$. HAVAL can produce a hash value of smaller sizes by using the output tailoring function. Because our attack target is the compression function, we omit the description for the padding and the output tailoring function.

The size of chaining variables is 256 bits. Inside the compression function, $M_{i}$ is divided into thirty-two 32 -bit message words $\left(m_{0}, m_{1}, \ldots, m_{31}\right)$. Three algorithms are prepared for HAVAL; 3-pass, 4-pass, and 5-pass. The number of rounds for 3-pass, 4-pass, and 5-pass are 3 rounds (96 steps), 4 rounds (128 steps), and 5 rounds (160 steps), respectively.

Let us denote a 256-bit state before step $j$ by $p_{j}$ and denote $p_{j}$ by eight 32 -bit variables $Q_{j-7}\left\|Q_{j-6}\right\| Q_{j-5}\left\|Q_{j-4}\right\| Q_{j-3}\left\|Q_{j-2}\right\| Q_{j-1} \| Q_{j}$. The step function $R_{j}$ computes $Q_{j+1}$ as follows:

$$
Q_{j+1} \leftarrow\left(Q_{j-7} \ggg 11\right)+\left(\Phi_{j}\left(\phi_{x, y}\left(Q_{j-6}, Q_{j-5}, \ldots, Q_{j}\right)\right) \ggg 7\right)+m_{\pi(j)}+k_{j},
$$

where $\phi_{x, y}$ is a word-wise rotation for $x$-pass HAVAL in round $y$ defined in Table 2, and $\pi(j)$ is shown in Table 3 .

\subsection{Technical Terminologies}

In this paper, we discuss differences of several computations. Let us consider the two computations $H_{i+1} \leftarrow \mathrm{CF}\left(H_{i}, M_{i}\right)$ and $H_{i+1}^{\prime} \leftarrow \mathrm{CF}\left(H_{i}^{\prime}, M_{i}^{\prime}\right)$. The message 
difference, input chaining-variable difference, and output difference are defined as $M_{i} \oplus M_{i}^{\prime}, H_{i} \oplus H_{i}^{\prime}$, and $H_{i+1} \oplus H_{i+1}^{\prime}$, respectively. Similarly, the difference of two computations is defined as XOR of corresponding states.

The transition of the difference of internal state is described by several terms such as differential path, differential trail, and differential characteristic. As far as we know, the term differential characteristic was firstly used in the context of the symmetric-key cryptography. However, in the context of the hash function analysis, the term differential path seems to be used more frequently e.g., [26]27. To follow this convention, in this paper, we use the term differential path.

When the input and output differences are fixed, we often consider all possible differential paths connecting them. A set of all possible differential paths is called a differential or multiple-paths. In this paper, we use the term differential.

\section{Related Work}

\subsection{Boomerang Attack}

The boomerang attack was proposed by Wagner [22] as a tool for attacking block-ciphers. The attack is a chosen-plaintext and adaptively chosen-ciphertext attack. It can be regarded as a type of the second-order differential attack. In this attack, the attacker divides the target cipher $E$ into two parts $E_{1}$ and $E_{2}$ such that $E(\cdot)=E_{2} \circ E_{1}(\cdot)$. Let us denote the differential for $E_{1}$ by $\Delta \rightarrow \Delta^{*}$ and for $E_{2}$ by $\nabla^{*} \rightarrow \nabla$. The differences $\Delta, \Delta^{*}, \nabla^{*}$, and $\nabla$ are chosen by the attacker at offline. The attack procedure is as follows;

1. The attacker first prepares a plaintext $P^{1}$ and compute $P^{2} \leftarrow P^{1} \oplus \Delta$.

2. $P^{1}$ and $P^{2}$ are passed to the encryption oracle and the attacker obtains the corresponding ciphertexts $C^{1}$ and $C^{2}$.

3. The attacker prepares the paired ciphertexts $C^{3} \leftarrow C^{1} \oplus \nabla$ and $C^{4} \leftarrow C^{2} \oplus \nabla$, and passes them to the decryption oracle.

4. Finally, the attacker checks whether or not $P^{3}$ and $P^{4}$ has the difference $\Delta$.

Assume that the probability for the differentials for $E_{1}$ and $E_{2}$ are $p$ and $q$, respectively. Then, $\operatorname{Pr}\left[P^{3} \oplus P^{4}=\Delta\right]$ is expressed as $p^{2} q^{2}$. In the end, we can conclude that if $E$ can be divided into two parts with a high-probability differential, the boomerang attack is very efficient.

For a long time, it was assumed that the differentials for $E_{1}$ and $E_{2}$ can be chosen independently. In 2009, Murphy pointed out that this was not sufficient, and discovered several examples of this case for DES and AES [12.

\subsection{Boomerang Distinguishers for Hash Functions}

In 2011, Biryukov et al. pointed out that the zero-sum distinguisher can be constructed by applying the boomerang attack on hash functions [3]. In the boomerang attack, $P^{1} \oplus P^{2}=\Delta$ and $P^{3} \oplus P^{4}=\Delta$. Therefore, $P^{1} \oplus P^{2} \oplus P^{3} \oplus P^{4}=$ $\Delta \oplus \Delta=0$. Similarly, $C^{1} \oplus C^{2} \oplus C^{3} \oplus C^{4}=\nabla \oplus \nabla=0$. Hence, by starting 
from a pair of plaintexts $P^{1}$ and $P^{2}$ such that $P^{1} \oplus P^{2}=\Delta$, the attacker finds a zero-sum quartet with a complexity of $\left(p^{2} q^{2}\right)^{-1}$. 3 considered the attack starting from the border state between $E_{1}$ and $E_{2}$, and optimized the attack by applying the message modification technique [26 27]. 3] also computed the complexity to find a zero-sum in a random function for $n$-bit output. They explained that by starting from two paired plaintexts (resp. ciphertexts) with pre-specified differences, the complexity to find a zero-sum quartet of ciphertexts (resp. plaintexts) is $2^{\frac{n}{2}}$. Note that [3] considered the differential path rather than the differential for their attack. In fact, to apply the message modification technique, considering a differential path is much easier than a differential. Also note that 3 . considered the observation by Murphy [12. They had to give up combining the best differential paths for $E_{1}$ and $E_{2}$ due to their dependency.

In 2011, Lamberger and Mendel independently applied the boomerang 4-sum for the SHA-2 compression function [10]. They claimed that the complexity for finding a 4-sum quartet in a random function without any limitation on the input is $2^{\frac{n}{3}}$ by using the generalized birthday attack 23 .

\subsection{Boomerang on Encryption Modes of MD4, MD5, and HAVAL}

Kim et al. applied the boomerang attack approach on the encryption modes of MD4, MD5, and HAVAL in the related-key model [9. They proposed boomerang distinguishers with $2^{6}$ queries for MD4, $2^{11.6}$ queries for MD5, and $2^{9.6}$ queries for 4-pass HAVAL. These attacks were verified by the machine experiment. Furthermore, they proposed differential paths for 5-pass HAVAL, and claimed that the boomerang distinguisher with $2^{61}$ queries was possible. They also claimed that this distinguisher was partially verified with an experiment on a reduced-round variant which was truncated for the first and the last several rounds.

Our attack framework is close to the one discussed in Sect. 3.2 but use the differential paths in 9] as a tool. In fact, we use the same paths as 9] for MD5 and 4-pass HAVAL. However, we need new differential paths for 5-pass HAVAL due to the flaw which we will point out in Sect. 5 .

\section{Summary of Boomerang Attack on Hash Function}

Because various techniques for the boomerang attack on hash functions are distributed in several papers, we summarize the attack framework. Therefore, most of the contents in this section were originally observed by [39]10].

The attack can be divided into five phases; 1$)$ message differences $(\Delta M)$, 2) differential paths and sufficient conditions $(D P), 3)$ contradiction between two paths $(C P), 4)$ message modification $(M M)$, and 5) amplified probability $(A P)$. We explain each phase with several observations specific to message-words permutation hash functions.

\subsection{Message Differences $(\Delta M)$}

A generic strategy for an $N_{R}$-round hash function is illustrated in Fig. [1. For $N_{R}=4$, the first two and last two rounds are regarded as $E_{1}$ and $E_{2}$, respectively. 


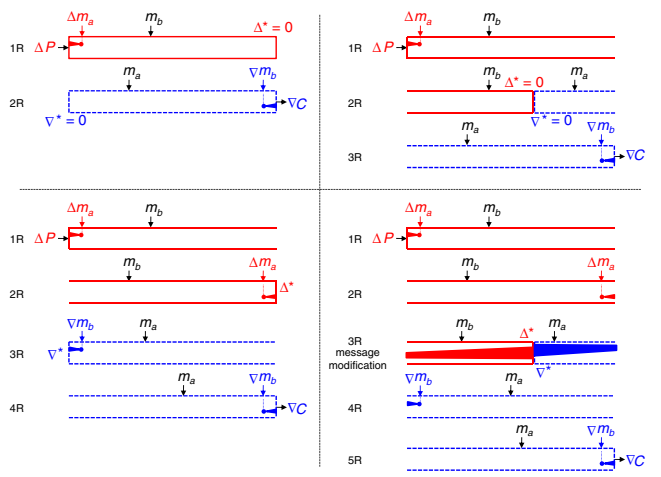

Fig. 1. Strategies for differential path

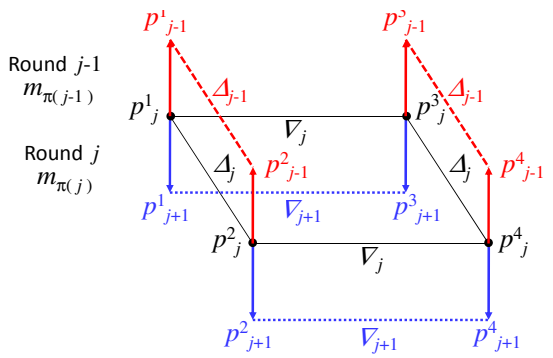

Fig. 2. Message search procedure

For $E_{1}$, we search for the message word which appear in an early step in the first round and in a late step in the second round. Then, the message difference is propagated until the beginning and end of $E_{1}$. The same strategy is applied for $E_{2}$. Because the differential paths for both of $E_{1}$ and $E_{2}$ are short, they are satisfied with high probability even without the message modification technique.

For $N_{R}=5$, we extend the differential paths for the 4-round attack by a half more round. As shown in Fig. 1 the paths become long and hard to satisfy by the naive search. Wang et al. showed that the differential path for one round can be satisfied for free by the message modification technique 26 27. Hence, with these techniques, 5 rounds can be attacked. In this paper, we denote the differential path between the end of round 2 and the beginning of round 4 by inside path, and the differential paths in round 1 and round 5 by outside paths.

\subsection{Differential Paths and Sufficient Conditions (DP)}

Based on the strategy in Fig. 1. we construct differential paths and conditions for chaining variables. These procedures are basically the same as the ones for previous collision attacks. We only list the differences of the path search procedure between the collision and boomerang attacks.

- If the feed-forward operation is performed by a modular addition $(H=$ $P \boxplus C)$, the attacker should introduce the additive difference among a quartet. in such a case, the difference for the quartet is defined as $\left(H^{4} \boxminus H^{3}\right) \boxminus\left(H^{2} \boxminus\right.$ $\left.H^{1}\right)=H^{1} \boxminus H^{2} \boxminus H^{3} \boxplus H^{4}$.

- The number of conditions must be minimized because setting $x$ more conditions will increase the complexity by a factor of $2^{2 x}$ rather than $2^{x}$.

- In the middle round, we apply the message modification, and thus even complicated paths can be satisfied. However, by taking into account the Phase $C P$, the paths should be simplified around the border of two paths. 
- If active-bit positions are concentrated around the MSB in both of $E_{1}$ and $E_{2}$ for the optimization, the risk of the contradiction of two paths will increase.

\subsection{Contradiction between Two Paths $(C P)$}

As Murphy pointed out [12], differential paths for $E_{1}$ and $E_{2}$ are not independent, and thus we need to check that any contradiction does not occur. As far as we know, no systematic method is known to check the contradiction. However, it can be said that the attacker at least needs to check the following conditions.

Condition 1. $E_{1}$ and $E_{2}$ do not require to fix the same bit to different values.

Condition 2. $E_{1}$ (resp. $E_{2}$ ) does not require to fix the value of an active bit for $E_{2}$ (resp. $E_{1}$ ).

The first case is obviously in contradiction. In the second case, even if the condition is satisfied between one pair, say $P^{1}$ and $P^{2}$, the condition is never satisfied for the other pair $P^{3}$ and $P^{4}$ due to the difference between $P^{1}$ and $P^{3}$.

As discussed in Sect. 4.2 if many bits are activated or active-bit positions are concentrated around MSB, the contradiction comes to occur more easily. Regarding HAVAL, due to the large word-size (32 bits) and the different rotation constants in forward and backward, the contradiction is less likely to occur. If the word size is smaller or if the similar rotation constants are used such as BLAKE, the contradiction seems to occur with a high probability.

If the contradiction occurs, rotating the path for either $E_{1}$ or $E_{2}$ by several bits may avoid the contradiction (though the efficiency becomes worse).

\subsection{Message Modification ( $M M$ )}

Let us denote a quartet of texts at step $j$ forming the boomerang structure by $\left(p_{j}^{1}, p_{j}^{2}, p_{j}^{3}, p_{j}^{4}\right)$. The difference for $E_{1}$ is denoted by $\Delta$, which is considered between $p_{1}$ and $p_{2}$ and between $p_{3}$ and $p_{4}$. The difference for $E_{2}$ is denoted by $\nabla$, which is considered between $p_{1}$ and $p_{3}$ and between $p_{2}$ and $p_{4}$. We call conditions on the path for $E_{1} \Delta$-conditions, and for $E_{2} \nabla$-conditions.

The message search procedure is described in Fig. 2. The attack starts from the state at the border between $E_{1}$ and $E_{2}$. Let us denote this step by $b$. First, we set a chaining-variables quartet $\left(p_{b}^{1}, p_{b}^{2}, p_{b}^{3}, p_{b}^{4}\right)$ so that both of $\Delta$ - and $\nabla$ conditions are satisfied. We then perform the backward computation for $E_{1}$ and forward computation for $E_{2}$ as shown in Alg. 11 and Alg. 2. respectively.

These procedures are computed until the inside path is ensured to be satisfied with probability of 1 . This often occurs before all message words are fixed by the above procedure. Therefore, towards the outside paths, we do as follows.

- Assume that several message-words are not determined even after the inside path are ensured. Then, we never modify the message-words and chainingvariables related to the inside path, and compute the outside paths by randomly choosing the message-words not used for the inside path.

This enables us to iterate the outside computation with keeping the inside path satisfied. Hence, the complexity for satisfying the inside path can be ignored. 

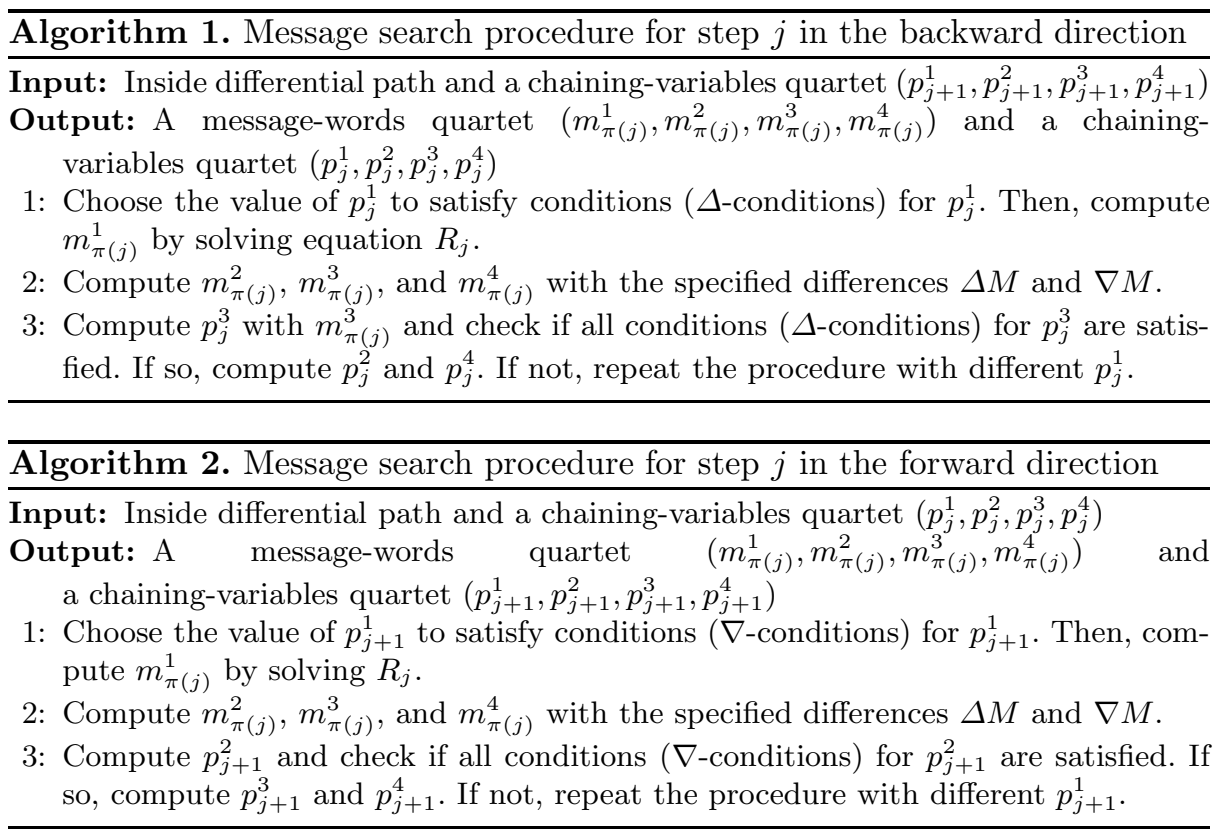

\subsection{Amplified Probability $(\boldsymbol{A P})$}

Amplified probability is the probability that each outside path results in the 4-sum. We consider the differential to estimate this probability. This is often estimated by an experiment. Alg. 3 shows how to compute the amplified probability $A P^{B a c k}$ for the first round (from step $j-1$ to step 0 ). The amplified probability for the final round $A P^{F o r}$ is similarly computed. Note that as long as the operation (usually either XOR or the modular addition) used to compute the 4-sum in this experiment and used in the feed-forward is identical, the success probability after the feed-forward is preserved as $A P^{B a c k} \times A P^{F o r}$.

\section{4-Sum Distinguisher on 5-Pass HAVAL}

We start from pointing out the flaw of the previous differential path (Sect. 5.1), and construct new differential paths (Sect. 5.2). We then explain the attack based on the discussion in Sect. 4 and finally show the experimental results.

\footnotetext{
Algorithm 3. Evaluation of the amplified probability

Input: Outside differential path

Output: Amplified probability of the outside differential path

1: Randomly choose a chaining-variables quartet $\left(p_{j}^{1}, p_{j}^{2}, p_{j}^{3}, p_{j}^{4}\right)$ and message-words used in steps $j-1$ to 0 with appropriate message differences $\Delta M$ and $\nabla M$.

2: Compute this quartet until step 0 and check whether the 4-sum is constructed.

3: Repeat the above for an enough amount of times, and calculate the probability.
} 
Table 4. Differential path and conditions for 5-pass HAVAL 9]. $e_{z}$ represents that only $z$-th bit has a difference.

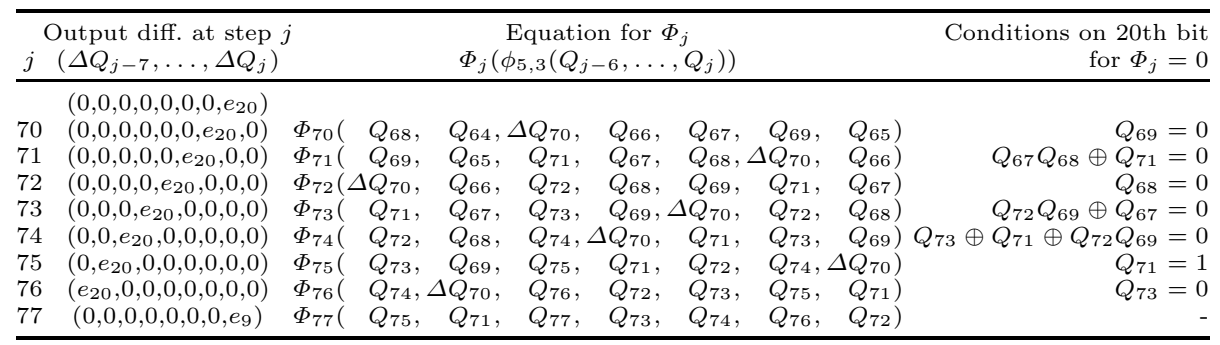

\subsection{Proving Flaw of Previous Differential Path}

We point out that the differential path for the third round in 9] cannot work. Note that the verifying experiment by [9] is for the reduced-round variants which are truncated for the first and the last several rounds [9, Sect. 4.2]. Hence, our claim does not contradict to the partial verification by 9 .

The differential path during 8 steps (steps 70-77) in the third round is shown in Table 4, The authors claimed that the path could be satisfied with a probability of $2^{-7}$. The necessary and sufficient condition for satisfying this path with a probability of $2^{-7}$ is that the difference in $Q_{70}$ will not propagate through $\Phi_{j}$. $\Phi_{j}$ for these steps is a bit-wise Boolean function expressed as

$$
\Phi_{j}\left(x_{6}, x_{5}, x_{4}, x_{3}, x_{2}, x_{1}, x_{0}\right)=x_{1} x_{2} x_{3} \oplus x_{1} x_{4} \oplus x_{2} x_{5} \oplus x_{3} x_{6} \oplus x_{0} x_{3} \oplus x_{0} .
$$

Conditions to achieve the path were not explained in $[9$. We first derive the necessary and sufficient conditions to achieve the path, which are shown in Table 4.

Proof. For steps 70, 75, and 76, we have conditions $Q_{69}=0, Q_{71}=1$, and $Q_{73}=0$. Then, the left-hand side of the condition for step 74 becomes $0 \oplus 1 \oplus$ $\left(Q_{72} \cdot 0\right)=1$, which contradicts to the condition that this value must be 0 .

We verified the above proof with a small experiment;

1. Randomly choose the values of $Q_{63}$ to $Q_{70}$ and $m_{\pi(70)}$ to $m_{\pi(77)}$.

2. Set $Q_{z}^{\prime} \leftarrow Q_{z}$ for $z=63,64, \ldots, 70$. Then compute $Q_{70}^{\prime} \leftarrow Q_{70} \oplus 0 \times 00100000$.

3. Compute until step 77 and check whether or not the path is satisfied.

With $2^{30}$ trials, the differential path in Table 4 was not satisfied. This contradicts to the claim in 9 , which the path is satisfied with a probability $2^{-7}$.

\subsection{Constructing New Differential Paths}

We reconstruct the attack based on the strategy explained Sect. 4.1 For Phase $\Delta M$, we confirmed that the message differences in [9] $\left(\Delta m_{2}=0 \times 80000000\right.$, 
Table 5. Boomerang path construction for 5-pass HAVAL

\begin{tabular}{|c|c|}
\hline & index for each round \\
\hline & 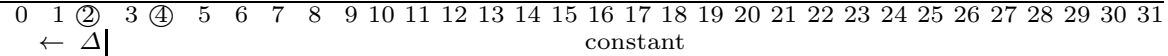 \\
\hline & $\begin{array}{cccccccccccccccccccc}7 & 16 & 0 & 23 & 20 & 22 & 1 & 10 & (4) \\
\text { constant }\end{array}$ \\
\hline & 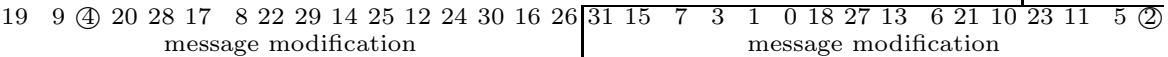 \\
\hline & 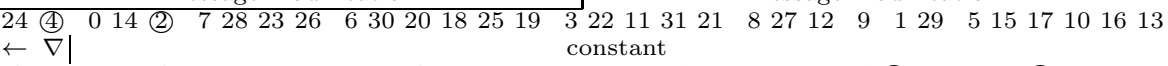 \\
\hline & 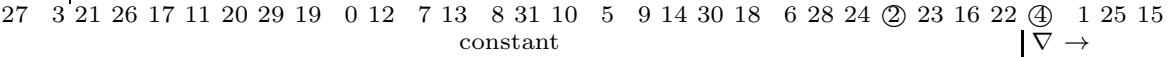 \\
\hline
\end{tabular}

$\Delta m_{x}=0$ for $x \neq 2, \nabla m_{4}=0 \times 80000000, \nabla m_{x}=0$ for $x \neq 4$ ) match the strategy in Fig. 1, and thus we use the same message differences. This is described in Table 5 .

For Phase $D P$, we need to specify how the differences will propagate to chaining variables. We describe our path search algorithm in Appendix A. The searched paths and conditions for chaining variables are given in Table 6 .

For Phase $C P$, the overlap of the conditions and active-bit positions in Table 6 must be checked. According to Table 6, the conditions 1 and 2 described in Sect. 4.3 are satisfied. Note that as long as the step function is similar to MD4, MD5, or HAVAL, the active bit positions and conditions for $\Delta$ and $\nabla$ tend to be different due to the asymmetric rotation constants in forward and backward directions. In fact, for all differential paths in [9] and ours, the best differential paths which were independently computed could be combined.

\subsection{Attack Procedure}

In Phase $M M$, we optimize the attack complexity based on the strategy in Sect. 4.4. The detailed procedure is given in Alg. 4.

As shown in Table 5 the inside path starts from step 60 and ends at step 97. Several words $\left(w_{2}, w_{25}, w_{31}, w_{27}, w_{24}, w_{4}\right)$ are used twice and we need a special attention. However, as shown in Table 6, conditions are set only on $Q_{58}$ to $Q_{93}$, and thus, the second-time use of these words outside of $Q_{58}$ to $Q_{93}$ always succeed for any value. Hence, these values are chosen for satisfying conditions for $Q_{58}$ to $Q_{93}$. After we satisfy all conditions for $Q_{58}$ to $Q_{93}, 4$ message words $w_{19}, w_{11}, w_{5}$, and $w_{2}$ are still unfixed. Therefore, we can iterate the outside path search without changing the inside path up to $2^{128}$ times, which is enough to satisfy the outside paths.

The complexity of the message modification for satisfying the inside path (up to Step 3 in Alg. 4) is negligible. Hence, the attack complexity is only the iterative computations for satisfying the outside paths (Steps 4-11 in Alg. 4). This complexity is evaluated by considering the amplified probability in Phase $A P$, which will be explained in the following section. 

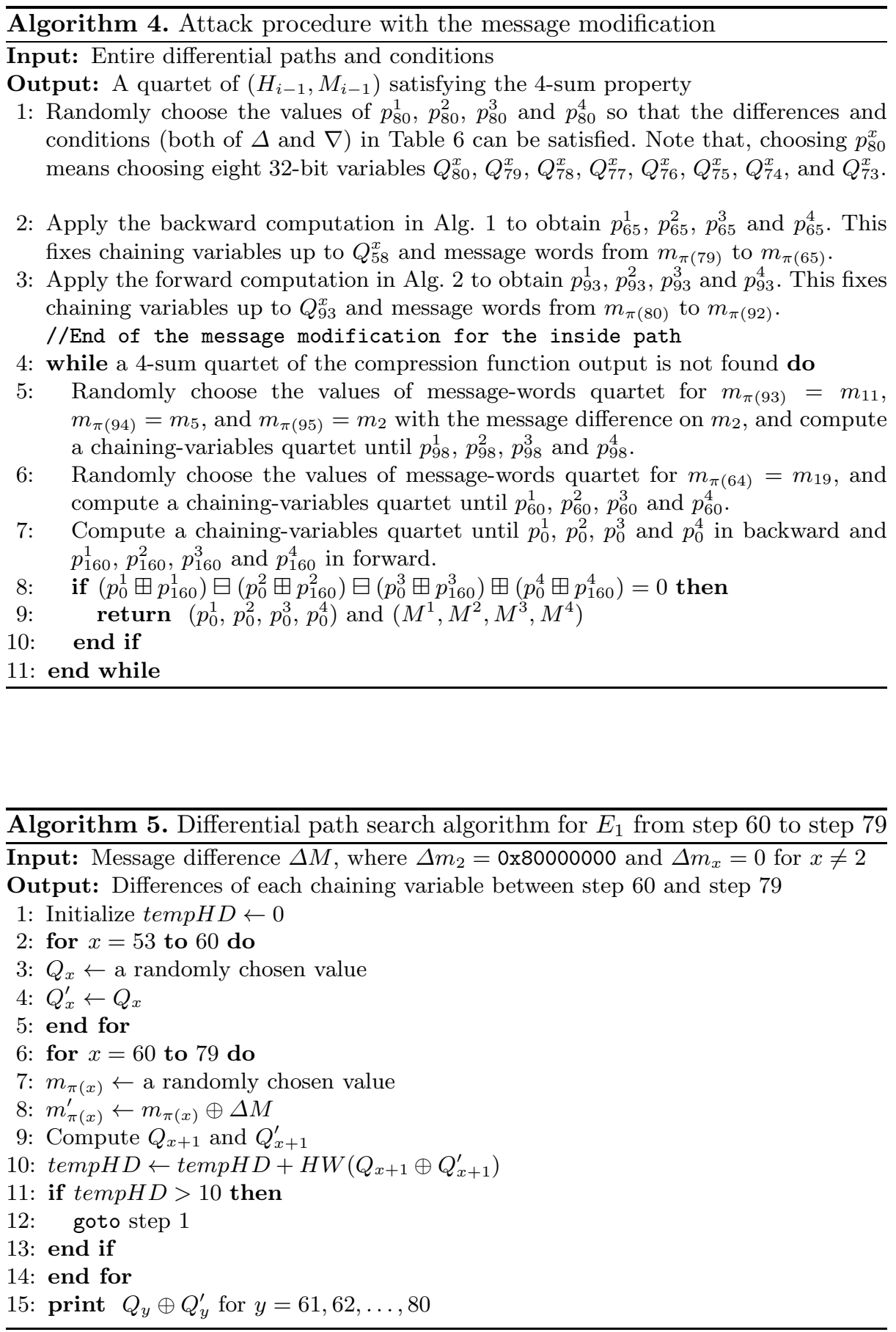
Table 6. New differential paths and conditions for 5-Pass HAVAL. $[z]=0,[z]=1$ are conditions on the value of $z$-th bit of the chaining variable. For the first and last several steps, we do not fix a particular difference for the amplified probability. The difference is considered in XOR. In some cases, we need conditions on the sign of the difference. $[z]=0+,[z]=1-$ mean the value is first fixed to 0 (resp. 1) and change to 1 (resp. 0 ) after the difference is inserted.

\begin{tabular}{|c|c|c|c|c|c|c|c|c|}
\hline \multicolumn{4}{|c|}{ Path for $E_{1}$ with $\Delta m_{2}=0 \times 80000000$} & \multicolumn{4}{|c|}{ Path for $E_{2}$ with $\nabla m_{4}=0 \times 80000000$} & \multirow[b]{2}{*}{$m_{\pi(j)}$} \\
\hline$j$ & $\Delta Q_{j}$ & Conditions on $Q_{j}$ & $\Delta m$ & $j$ & $\nabla Q_{j}$ & Conditions on $Q_{j}$ & $\nabla m$ & \\
\hline-7 & $A P$ & $A P$ & & -7 & & & & $m_{1}$ \\
\hline-6 & $A P$ & $A P$ & & -6 & & & & $m_{0}$ \\
\hline-5 & $A P$ & $A P$ & $0 \times 80000000$ & -5 & & & & $m_{2}$ \\
\hline-4 & & & & -4 & & & & $m_{3}$ \\
\hline 52 & 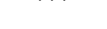 &.. & & 52 & . & 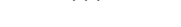 & $\cdots$ & $m_{13}$ \\
\hline 53 & & & $0 \times 80000000$ & 53 & & & & $m_{2}$ \\
\hline 54 & & & & 54 & & & & $m_{25}$ \\
\hline 55 & & & & 55 & & & & $m_{31}$ \\
\hline 56 & & & & 56 & & & & $m_{27}$ \\
\hline 57 & & & & 57 & & & & $m_{19}$ \\
\hline 58 & & {$[31]=0$} & & 58 & & & & $m_{9}$ \\
\hline 59 & & {$[31]=0$} & & 59 & & & & $m_{4}$ \\
\hline 60 & & {$[31]=0$} & & 60 & & & & $m_{20}$ \\
\hline 61 & $0 \times 80000000$ & & & 61 & & & & $m_{28}$ \\
\hline 62 & & {$[31]=0$} & & 62 & & & & $m_{17}$ \\
\hline 63 & & {$[31]=0$} & & 63 & & & & $m_{8}$ \\
\hline 64 & & {$[31,24]=0$} & & 64 & & & & $m_{22}$ \\
\hline 65 & & {$[24]=0$} & & 65 & & & & $m_{29}$ \\
\hline 66 & & {$[24,20]=0$} & & 66 & & & & $m_{14}$ \\
\hline 67 & $0 \times 01000000$ & {$[20]=0$} & & 67 & & & & $m_{25}$ \\
\hline 68 & & {$[24,20]=0$} & & 68 & & & & $m_{12}$ \\
\hline 69 & $0 \times 00100000$ & {$[24]=0$} & & 69 & & & & $m_{24}$ \\
\hline 70 & & {$[24,20,17]=0$} & & 70 & & & & $m_{30}$ \\
\hline 71 & & {$[20,17]=0$} & & 71 & & & & $m_{16}$ \\
\hline 72 & & {$[24,20,17]=0$} & & 72 & & & & $m_{26}$ \\
\hline 73 & $0 \times 00020000$ & {$[17]=1-$} & & 73 & & & & \\
\hline 74 & & {$[20,17]=0$} & & 74 & $0 \times 00000001$ & {$[0]=1-$} & & \\
\hline 75 & & {$[17,9]=0$} & & 75 & & {$[18]=0$} & & \\
\hline 76 & & {$[17,9]=0$} & & 76 & & {$[18]=0$} & & start \\
\hline 77 & $0 \times 00000200$ & {$[9]=0+$} & & 77 & & {$[18,0]=0$} & & step \\
\hline 78 & & {$[17,10,9]=0$} & & 78 & $0 \times 00040000$ & {$[21]=0,[18]=0+$} & & \\
\hline $\begin{array}{l}79 \\
80\end{array}$ & $0 \times 00000400$ & {$[10]=1-$} & & $\begin{array}{l}79 \\
80\end{array}$ & & {$[21]=0,[18]=1$} & & \\
\hline 81 & & & & 81 & & {$[21,18,14]=0$} & & $m_{31}$ \\
\hline 82 & & & & 82 & $0 \times 00200000$ & {$[14]=0$} & & $m_{15}$ \\
\hline 83 & & & & 83 & & {$[21]=1,[14]=0$} & & $m_{7}$ \\
\hline 84 & & & & 84 & $0 \times 00004000$ & {$[21]=0$} & & $m_{3}$ \\
\hline 85 & & & & 85 & & {$[21]=0,[14]=1$} & & $m_{1}$ \\
\hline 86 & & & & 86 & & {$[14]=0$} & & $m_{0}$ \\
\hline 87 & & & & 87 & & {$[14,10]=0$} & & $m_{18}$ \\
\hline 88 & & & & 88 & & {$[10]=0$} & & $m_{27}$ \\
\hline 89 & & & & 89 & & {$[10]=0$} & & $m_{13}$ \\
\hline 90 & & & & 90 & $0 \times 00000400$ & & & $m_{6}$ \\
\hline 91 & & & & 91 & & {$[10]=1$} & & $m_{21}$ \\
\hline 92 & & & & 92 & & {$[10]=1$} & & $m_{10}$ \\
\hline 93 & & & & 93 & & {$[10]=1$} & & $m_{23}$ \\
\hline 94 & & & & 94 & & & & $m_{11}$ \\
\hline 95 & & & & 95 & & & & $m_{5}$ \\
\hline 96 & & & & 96 & & & & $m_{2}$ \\
\hline 97 & & & & 97 & & & & $m_{24}$ \\
\hline 98 & & & & 98 & & & $0 \times 80000000$ & $m_{4}$ \\
\hline 99 & & & & 99 & & & & $m_{0}$ \\
\hline & $\cdots$ & $\cdots$ & $\cdots$ & & $\cdots$ & $\cdots$ & & $\cdots$ \\
\hline 157 & & & & $\begin{array}{l}150 \\
157\end{array}$ & $0 \times 80000000$ & $A P$ & $0 \times 80000000$ & $\left.\right|_{m} ^{m} 22$ \\
\hline 158 & & & & 158 & $A P$ & $A P$ & & $m_{1}$ \\
\hline 159 & & & & 159 & $A P$ & $A P$ & & $m_{25}$ \\
\hline 160 & & & & 160 & $A P$ & $A P$ & & $m_{15}$ \\
\hline
\end{tabular}


Table 7. Experimental results for the amplified probability

\begin{tabular}{lccc}
\hline Direction & Number of trials & Number of obtained 4-sums & Amplified probability \\
\hline Back & $1,000,000$ & 53,065 & $2^{-4.24}$ \\
For & $1,000,000$ & 37,623 & $2^{-4.73}$ \\
Total & $1,000,000$ & 1,975 & $2^{-8.98}$ \\
\hline
\end{tabular}

Table 8. An example of the boomerang quartet for the full 5-pass HAVAL

\begin{tabular}{|c|c|}
\hline$H_{i}^{1}$ & $6 a d 6913 \mathrm{~b}$ 0x52831497 0x42e2afea 0x042171e8 0x05c66540 0xf6308a5d 0x69b242bb 0x \\
\hline$M_{i}^{1}$ & 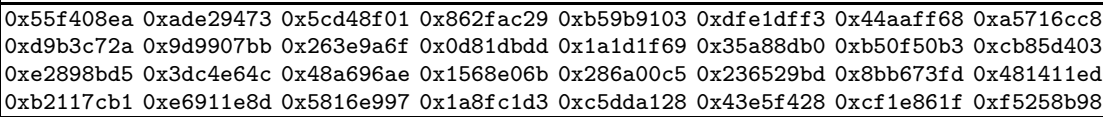 \\
\hline$H_{i+1}^{1}$ & 550b484bf 0x9d28c720 0xc2a5ab4d 0x5aec2d4b 0x63659cae 0x0023f316 0xa02276be 0x \\
\hline 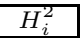 & 0x6ad6913b 0x52831497 0x42e2afea 0x042171e8 0x05c66540 0xf6308e5d 0x69b242bb 0x \\
\hline$\overline{M_{i}^{2}}$ & 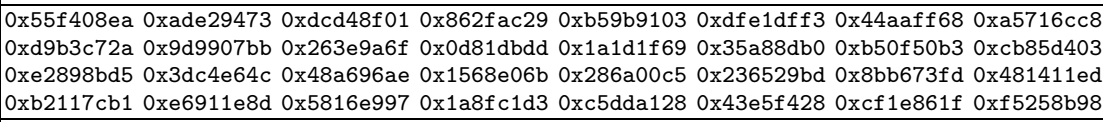 \\
\hline$\overline{H_{i+1}^{2}}$ & 0xfa15769c 0x6ed1b19a 0x405b263b 0x57cd6359 0xd8688750 0xcdc3c9d3 0xa3dc7fd8 0x2e59f283 \\
\hline 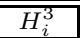 & xb70b5251 0x851d041a 0x7a5f5fad 0x98626bb1 0x9d739cbc 0x67bc3181 0xe48e4cac 0xe \\
\hline$M_{i}^{3}$ & 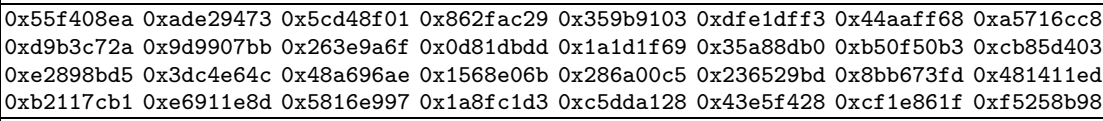 \\
\hline$H_{i+1}^{3}$ & 0x9ce945d5 0xcfc2b6a3 0xfa225b10 0xef2d2714 0x7b12d42a 0x71af9a3a 0x1afe80af 0xdbbd87cb \\
\hline 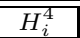 & 0xb70b5251 0x851d041a 0x7a5f5fad 0x98626bb1 0x9d739cbc 0x \\
\hline$M_{i}^{4}$ & 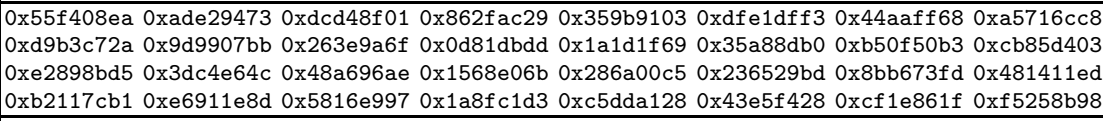 \\
\hline$H_{i+1}^{4}$ & 0x464a37b2 0xa16ba11d 0x77d7d5fe 0xec0e5d22 0xf015becc 0x3f4f70f7 0x1eb889c9 0x1f617eca \\
\hline & $8000000000 \times 000000000 \times 000000000 \times 000000000 \times 000000000 \times 000000000 \times 000000000 x$ \\
\hline
\end{tabular}

Table 9. An example of the boomerang quartet for MD5

\begin{tabular}{c|l}
\hline$H_{i}^{1}$ & 0x7ad51bee 0x68a07529 0x5369e5f1 0x62f52251 \\
\hline$M_{i}^{1}$ & $\begin{array}{l}\text { 0x58df0f5e 0x678b3525 0x03105c08 0xa068f82a 0x21ead339 0xe6e2ea9c 0x5cf986e1 0x9890fd27 } \\
\text { 0xcf8a438f 0x2cecb915 0x44935dfe 0xf06f103f 0x72d5b376 0x9688dfed 0x7b2ae2f6 0xe9256628 }\end{array}$ \\
\hline$H_{i+1}^{1}$ & 0x1de7b79a 0x6e573e2a 0x0ef900e3 0xc72985ef \\
\hline \hline$H_{i}^{2}$ & 0xfad51bee 0x68a07529 0xd369e5f1 0xe2f52251 \\
\hline$M_{i}^{2}$ & $\begin{array}{l}\text { 0x58df0f5e 0x678b3525 0x83105c08 0xa068f82a 0x21ead339 0xe6e2ea9c 0x5cf986e1 0x9890fd27 } \\
\text { 0xcf8a438f 0x2cecb915 0x44935dfe 0xf06f103f 0x72d5b376 0x9688dfed 0x7b2ae2f6 0xe9256628 }\end{array}$ \\
\hline$H_{i+1}^{2}$ & 0x03d5ae50 0x722a5685 0x361b13a1 0x75a3a89d \\
\hline \hline$H_{i}^{3}$ & 0x97e364fe 0xb191e24c 0xdec0361f 0x6a8d3d9f \\
\hline$M_{i}^{3}$ & 0x58df0f5e 0x678b3525 0x03105c08 0xa068f82a 0x21ead339 0xe6e2ea9c 0x5cf986e1 0x9890fd27 \\
\hline$H_{i+1}^{3}$ & 0x3f8a438f 0x2cecb915 0x44935dfe 0x706f103f 0x72d5b376 0x9688dfed 0x7b2ae2f6 0xe9256628 \\
\hline \hline$H_{i}^{4}$ & 0x17e364fe 0xb191e24c 0x5ec0361f 0xea8d3d9f \\
\hline$M_{i}^{4}$ & 0x58df0f5e 0x678b3525 0x83105c08 0xa068f82a 0x21ead339 0xe6e2ea9c 0x5cf986e1 0x9890fd27 \\
\hline$H_{i+1}^{4}$ & 0xcf8a438f 0x2cecb915 0x44935dfe 0x706f103f 0x72d5b376 0x9688dfed 0x7b2ae2f6 0xe9256628 \\
\hline \hline
\end{tabular}

\begin{tabular}{c|c|c|}
\hline 4-sum & $0 \times 00000000$ 0x00000000 0x00000000 0x00000000
\end{tabular} 
Table 10. An example of the boomerang quartet for 3-pass HAVAL

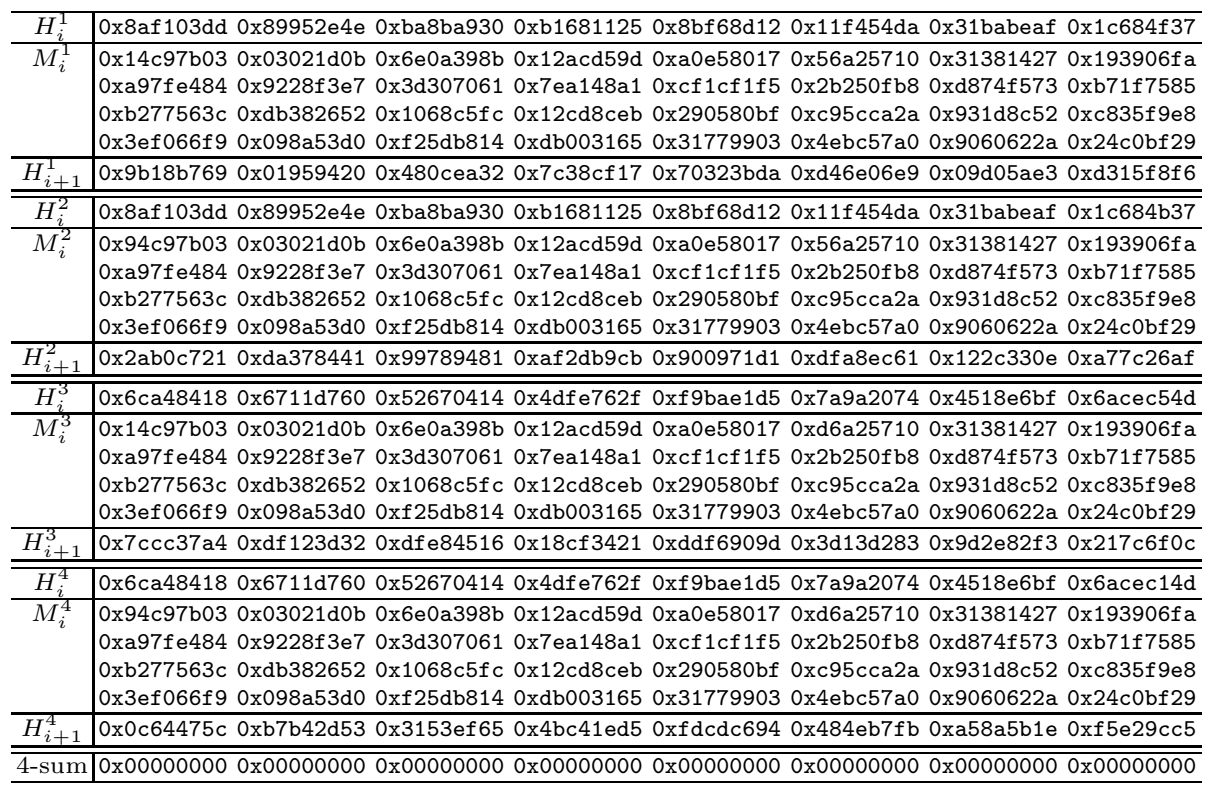

Table 11. An example of the boomerang quartet for 4-pass HAVAL

\begin{tabular}{|c|c|}
\hline$H_{i}^{1}$ & x564187b3 0x5af775fb 0x10136ca0 0x8a9ffa0c 0x3edeecd0 0xd2e74f6c 0x \\
\hline$M_{i}^{1}$ & 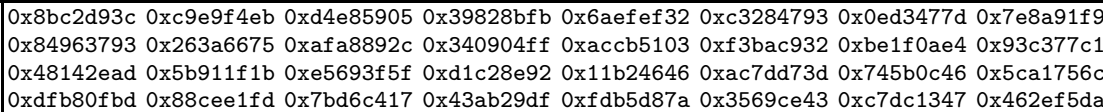 \\
\hline$H_{i+1}^{1}$ & x910e3d63 0x83c406ec 0x464230f7 0x3bfc4d84 0x14fddff2 0x5092bf5f 0x07cd2ad3 0x \\
\hline$\overline{H_{i}^{2}}$ & $564187 \mathrm{~b} 3$ 0x5af775fb 0x10136ca0 0x8a9ffa0c 0x3edeecd0 0xd2e74b6c 0x15572f1c 0x \\
\hline$M_{i}^{2}$ & 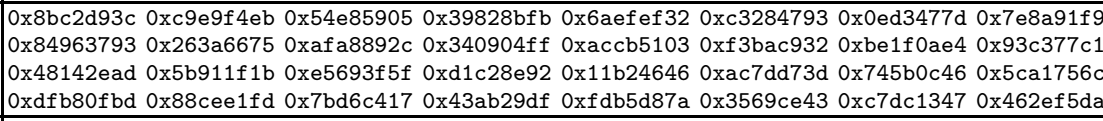 \\
\hline$H_{i+1}^{2}$ & 0xe6b6ce20 0x3260de7a 0x681aa45d 0x277995cd 0x9d4959bc 0xaec251c2 0x41446efa 0x75c \\
\hline$H_{i}^{3}$ & 0xaf8abf6d 0xd2aafd4c 0xc7f01506 0xfd258be0 0x299edd95 0xa561cfbc 0x61175f52 0x \\
\hline$M_{i}^{3}$ & 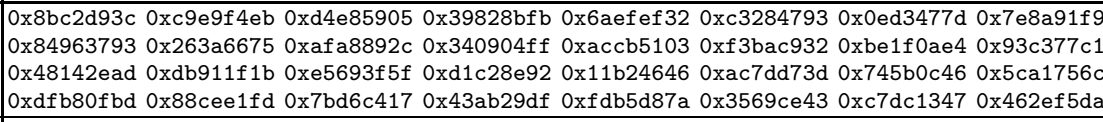 \\
\hline$H_{i+1}^{3}$ & 0xea57751d 0xfb778e3d 0xfe1ed95d 0xae81df58 0x7fbdd0b7 0x230d3faf 0x538d1b09 0xad631e53 \\
\hline$H_{i}^{4}$ & 0xaf8abf6d 0xd2aafd4c 0xc7f01506 0xfd258be0 0x299edd95 0xa561cbbc 0x61171f52 0x \\
\hline$M_{i}^{4}$ & 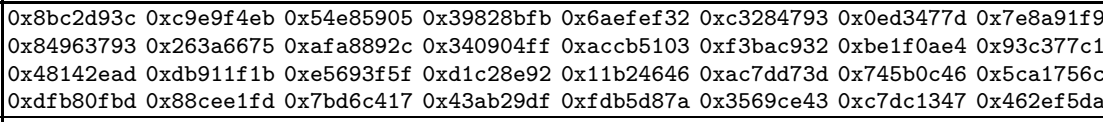 \\
\hline$H_{i+1}^{4}$ & 0x400005da 0xaa1465cb 0x1ff74cc3 0x99ff27a1 0x08094a81 0x813cd212 0x8d045f30 0xf1716669 \\
\hline tur & renongonos \\
\hline
\end{tabular}




\subsection{Experimental Results}

By following the algorithm in Alg. 3, we evaluated the amplified probability for the first and last several rounds. The results are shown in Table 7 . According to our experiments, $A P^{B a c k}=2^{-4.24}, A P^{F o r}=2^{-4.73}$, and the entire success probability is $2^{-8.98}$, which matches $A P^{B a c k} \times A P^{F o r}=2^{-8.97}$. The attack complexity is for $2^{8.98}$ iterations of Steps $4-11$ in Alg. 4. Because we compute quartets, the complexity is approximately $2^{11}\left(\approx 4 \times 2^{8.98}\right)$ compression function computations. Finally, we implemented our 4-sum distinguisher on 5-pass HAVAL. An example of the generated 4-sum quartet is presented in Table 8.

\section{Concluding Remarks}

We studied the boomerang attack approach on hash functions. We proved that the previous differential path on 5-pass HAVAL contained a flaw. We then constructed the new path and proposed the 4-sum distinguisher on the compression function with a complexity of approximately $2^{11}$ computations. We implemented the attack and showed an example of the 4-sum quartet. As far as we know, this is the first feasible result on the full compression function of 5-pass HAVAL.

\section{References}

1. Aoki, K., Sasaki, Y.: Preimage Attacks on One-Block MD4, 63-Step MD5 and More. In: Avanzi, R.M., Keliher, L., Sica, F. (eds.) SAC 2008. LNCS, vol. 5381, pp. 103-119. Springer, Heidelberg (2009)

2. Aumasson, J.-P., Meier, W., Mendel, F.: Preimage Attacks on 3-Pass HAVAL and Step-Reduced MD5. In: Avanzi, R.M., Keliher, L., Sica, F. (eds.) SAC 2008. LNCS, vol. 5381, pp. 120-135. Springer, Heidelberg (2009)

3. Biryukov, A., Nikolić, I., Roy, A.: Boomerang Attacks on BLAKE-32. In: Joux, A. (ed.) FSE 2011. LNCS, vol. 6733, pp. 218-237. Springer, Heidelberg (2011)

4. den Boer, B., Bosselaers, A.: Collisions for the Compression Function of MD-5. In: Helleseth, T. (ed.) EUROCRYPT 1993. LNCS, vol. 765, pp. 293-304. Springer, Heidelberg (1994)

5. Dobbertin, H.: The Status of MD5 after a Recent Attack. CryptoBytes The technical newsletter of RSA Laboratories, a division of RSA Data Security, Inc. 2(2) (Summer 1996)

6. Guo, J., Ling, S., Rechberger, C., Wang, H.: Advanced Meet-in-the-Middle Preimage Attacks: First Results on Full Tiger, and Improved Results on MD4 and SHA-2. In: Abe, M. (ed.) ASIACRYPT 2010. LNCS, vol. 6477, pp. 56-75. Springer, Heidelberg (2010)

7. Joux, A., Peyrin, T.: Hash Functions and the (Amplified) Boomerang Attack. In: Menezes, A. (ed.) CRYPTO 2007. LNCS, vol. 4622, pp. 244-263. Springer, Heidelberg (2007)

8. Kim, J.-S., Biryukov, A., Preneel, B., Lee, S.-J.: On the Security of Encryption Modes of MD4, MD5 and HAVAL. In: Qing, S., Mao, W., López, J., Wang, G. (eds.) ICICS 2005. LNCS, vol. 3783, pp. 147-158. Springer, Heidelberg (2005) 
9. Kim, J., Biryukov, A., Preneel, B., Lee, S.: On the Security of Encryption Modes of MD4, MD5 and HAVAL. Cryptology ePrint Archive, Report 2005/327 (2005); In: Qing, S., Mao, W., López, J., Wang, G. (eds.) ICICS 2005. LNCS, vol. 3783, pp. 147-158. Springer, Heidelberg (2005)

10. Lamberger, M., Mendel, F.: Higher-Order Differential Attack on Reduced SHA256. Cryptology ePrint Archive, Report 2011/037 (2011), http://eprint.iacr.org/2011/037

11. Leurent, G.: MD4 is Not One-Way. In: Nyberg, K. (ed.) FSE 2008. LNCS, vol. 5086, pp. 412-428. Springer, Heidelberg (2008)

12. Murphy, S.: The Return of the Cryptographic Boomerang. IEEE Transactions on Information Theory 57(4), 2517-2521 (2011)

13. Rivest, R.L.: The MD4 Message Digest Algorithm. In: Menezes, A., Vanstone, S.A. (eds.) CRYPTO 1990. LNCS, vol. 537, pp. 303-311. Springer, Heidelberg (1991), also appeared in RFC 1320 http://www.ietf.org/rfc/rfc1320.txt

14. Rivest, R.L.: Request for Comments 1321: The MD5 Message Digest Algorithm. The Internet Engineering Task Force (1992)

15. Sakai, Y., Sasaki, Y., Wang, L., Ohta, K., Sakiyama, K.: Preimage Attacks on 5- Pass HAVAL Reduced to 158-Steps and One-Block 3-Pass HAVAL. Industrial Track of ACNS 2011 (2011)

16. Sasaki, Y., Aoki, K.: Preimage Attacks on 3, 4, and 5-Pass HAVAL. In: Pieprzyk, J.P. (ed.) ASIACRYPT 2008. LNCS, vol. 5350, pp. 253-271. Springer, Heidelberg (2008)

17. Sasaki, Y., Aoki, K.: Finding Preimages in Full MD5 Faster than Exhaustive Search. In: Joux, A. (ed.) EUROCRYPT 2009. LNCS, vol. 5479, pp. 134-152. Springer, Heidelberg (2009)

18. Sasaki, Y., Wang, L., Ohta, K., Kunihiro, N.: New Message Difference for MD4. In: Biryukov, A. (ed.) FSE 2007. LNCS, vol. 4593, pp. 329-348. Springer, Heidelberg (2007)

19. Suzuki, K., Kurosawa, K.: How to Find Many Collisions of 3-Pass HAVAL. In: Miyaji, A., Kikuchi, H., Rannenberg, K. (eds.) IWSEC 2007. LNCS, vol. 4752, pp. 428-443. Springer, Heidelberg (2007)

20. U.S. Department of Commerce, National Institute of Standards and Technology: Federal Register Vol. 72, No. 212/Friday, November 2, 2007/Notices (2007)

21. Van Rompay, B., Biryukov, A., Preneel, B., Vandewalle, J.: Cryptanalysis of 3-Pass HAVAL. In: Laih, C.-S. (ed.) ASIACRYPT 2003. LNCS, vol. 2894, pp. 228-245. Springer, Heidelberg (2003)

22. Wagner, D.: The Boomerang Attack. In: Knudsen, L.R. (ed.) FSE 1999. LNCS, vol. 1636, pp. 156-170. Springer, Heidelberg (1999)

23. Wagner, D.: A Generalized Birthday Problem. In: Yung, M. (ed.) CRYPTO 2002. LNCS, vol. 2442, pp. 288-303. Springer, Heidelberg (2002)

24. Wang, X., Feng, D., Yu, X.: An Attack on Hash Function HAVAL-128. Science in China (Information Sciences) 48(5), 545-556 (2005)

25. Wang, X., Lai, X., Feng, D., Chen, H., Yu, X.: Cryptanalysis of the Hash Functions MD4 and RIPEMD. In: Cramer, R. (ed.) EUROCRYPT 2005. LNCS, vol. 3494, pp. 1-18. Springer, Heidelberg (2005)

26. Wang, X., Yin, Y.L., Yu, H.: Finding Collisions in the Full SHA-1. In: Shoup, V. (ed.) CRYPTO 2005. LNCS, vol. 3621, pp. 17-36. Springer, Heidelberg (2005)

27. Wang, X., Yu, H.: How to Break MD5 and Other Hash Functions. In: Cramer, R. (ed.) EUROCRYPT 2005. LNCS, vol. 3494, pp. 19-35. Springer, Heidelberg (2005) 
28. Wang, Z., Zhang, H., Qin, Z., Meng, Q.: Cryptanalysis of 4-Pass HAVAL. Crptology ePrint Archive, Report 2006/161 (2006)

29. Xie, T., Liu, F., Feng, D.: Could the 1-MSB Input Difference be the Fastest Collision Attack for MD5? Cryptology ePrint Archive, Report 2008/391 (2008)

30. Yoshida, H., Biryukov, A., De Cannière, C., Lano, J., Preneel, B.: Non-Randomness of the Full 4 and 5-Pass HAVAL. In: Blundo, C., Cimato, S. (eds.) SCN 2004. LNCS, vol. 3352, pp. 324-336. Springer, Heidelberg (2005)

31. Yu, H., Wang, X., Yun, A., Park, S.: Cryptanalysis of the Full HAVAL with 4 and 5 Passes. In: Robshaw, M.J.B. (ed.) FSE 2006. LNCS, vol. 4047, pp. 89-110. Springer, Heidelberg (2006)

32. Zheng, Y., Pieprzyk, J., Seberry, J.: HAVAL - One-Way Hashing Algorithm with Variable Length of Output. In: Zheng, Y., Seberry, J. (eds.) AUSCRYPT 1992. LNCS, vol. 718, pp. 83-104. Springer, Heidelberg (1993)

\section{A Differential Path Search Algorithm for 5-Pass HAVAL}

Our path search algorithm is semi-automated and minimizes the Hamming distance of the entire inside path. We independently searched for the path for $E_{1}($ steps $60-79)$ with $\Delta m_{2}=0 \times 80000000$ and path for $E_{2}$ (steps $98-73$ ) with $\nabla m_{4}=0 \times 80000000$. Conditions and contradiction of two paths were later checked by hand. We only explain the algorithm for $E_{1}$ in Alg. [5] $H W(\cdot)$ returns the Hamming weight of the input variable.

After an enough number of iterations of Alg. 5] we obtained the path in Table 6 whose tempHD is 6 .

\section{B Examples of Boomerang Quartet}

The differential paths in 9] can be used to construct a 4-sum on the compression function. We show the generated 4-sums for MD5, 3-pass HAVAL, and 4-pass HAVAL. The amplified probability to satisfy the entire path is approximately $2^{-8}$ for MD5, $2^{-2}$ for 3 -pass HAVAL, and $2^{-9}$ for 4 -pass HAVAL. 